\title{
Mapeamento Tecnológico para o Desenvolvimento de um Sistema de Avaliação de Riscos/Impactos Ambientais: uma ferramenta para sustentabilidade e desenvolvimento territorial
}

\author{
Technological Mapping for the Development of a System for Assessing \\ Environmental Impacts Based on the Leopold Matrix
}

\author{
Roberto Tenório Figueiredo ${ }^{1}$ \\ Vivianni Marques Leite dos Santos ${ }^{2}$ \\ Jorge Luis Cavalcanti Ramos ${ }^{2}$ \\ ${ }^{1}$ Faculdade de Petrolina, Petrolina, PE, Brasil \\ ${ }^{2}$ Universidade Federal do Vale do São Francisco, Petrolina, PE, Brasil
}

\begin{abstract}
Resumo
A conservação do meio ambiente é prioridade mundial e passou a ser, além de um fator vital, uma questão legal, com leis para os projetistas com atividades potencialmente impactantes ao meio ambiente. A legislação obriga que seja feita uma avaliação de impactos ambientais criteriosa, inclusive confeccionando relatórios técnicos específicos que detalham a análise feita por profissionais especialistas. O objetivo deste trabalho é elaborar um Roadmap Tecnológico a partir do levantamento das famílias de patentes, registros de softwares e trabalhos publicados alusivos à avaliação de impactos ambientais baseados na Matriz de Leopold. É feito o levantamento em diversas plataformas de busca e seus resultados são compilados e analisados. A conclusão é pela viabilidade do sistema de avaliação de impactos ambientais com benefícios a todos os agentes envolvidos em projetos, disponibilizando a informação de maneira precisa, completa e rápida e dando o suporte necessário à tomada de decisão.
\end{abstract}

Palavras-chave: Avaliação de Impactos Ambientais. Matriz de Leopold. Tomada de Decisão.

\begin{abstract}
Environmental conservation is a world priority and, in addition to being a vital factor, has become a legal issue, with laws for project designers with activities potentially impacting the environment. The legislation requires a careful environmental impacts assessment to be carried out, including the preparation of specific technical reports, which detail the analysis made by specialist professionals. The objective of this work is to elaborate a Technological Roadmap from the survey of patent families, software records and published works referring to the assessment of environmental impacts based on the Leopold Matrix. The survey is made on several search platforms and its results are compiled and analyzed. The conclusion indicates the feasibility of the environmental impacts assessment system with benefits for all agents involved in projects, making information available in a precise, complete and fast way, providing the necessary support for decision making.
\end{abstract}

Keywords: Environmental Impact Assessment. Leopold Matrix. Decision Making.

Área Tecnológica: Desenvolvimento. Inovação. Prospecção Tecnológica. 


\section{Introdução}

Depois da Segunda Guerra Mundial, pesquisadores de várias partes do mundo realizaram estudos que comprovaram os inúmeros impactos ambientais negativos a partir da intervenção humana no meio ambiente global. Esses estudos contêm registros que apontam a necessidade de realizar o desenvolvimento socioeconômico considerando análise prévia dos prejuízos ao planeta, com o intuito de minimizá-los ou de excluí-los desde seu planejamento. Nesse sentido, a Organização das Nações Unidas (ONU) realizou, em junho de 1972, a I Conferência Mundial de Meio Ambiente em Estocolmo, na Suécia. Nessa Conferência, foi criada uma ferramenta para auxiliar o planejamento de projetos com atividade potencialmente causadora de significativa degradação do meio ambiente, a Avaliação de Impactos Ambientais (AIA) (MMA, 2009).

Neste texto, considera-se impacto ambiental como:

[...] qualquer alteração das propriedades físicas, químicas e biológicas do meio ambiente, causada por qualquer forma de matéria ou energia resultante das atividades humanas que, direta ou indiretamente, afetam: I - a saúde, a segurança e o bem-estar da população; II - as atividades sociais e econômicas; III - a biota; IV - as condições estéticas e sanitárias do meio ambiente; $\mathrm{V}$ - a qualidade dos recursos ambientais. (CONAMA, 1986)

O objetivo principal da AIA, segundo Batista et al. (2017), é elencar todos os fatores ambientais inerentes a um projeto para que as decisões tomadas sobre esse projeto levem em consideração tais fatores, ou seja, permitir que sejam tomadas ações preventivas, quando realizadas na fase de projeto, e também corretivas, quando realizadas para um projeto em execução ou mesmo finalizado.

A Avaliação de Impactos Ambientais, segundo Enríquez-de-Salamanca (2018), é uma reunião de procedimentos que tem por objetivo identificar e classificar potenciais impactos que uma atividade pode causar ao meio ambiente, prevendo a abrangência e os prejuízos dessas atividades impactantes. A AIA é feita por técnicos e especialistas e auxilia os projetistas a criarem projetos que não agridam o meio ambiente (SANGUINETTO, 2011).

Os elementos que compõem a AIA trazem para o projeto seus possíveis impactos ambientais, antes da tomada de decisão sobre sua implantação, ainda em fase de planejamento. A participação de terceiros, afetados pelo projeto, é um componente importante nessa avaliação, fazendo da AIA uma ferramenta participativa de gestão ambiental (JAY et al., 2007).

Apesar da diferença entre risco e impacto, visto que o risco está associado à previsão de ocorrência de um possível impacto e o impacto, em si, já configura sua ocorrência (DAGNINO; CARPI JUNIOR, 2007), neste texto, a ferramenta de análise pode ser aplicada a ambos, logo sua aplicação será discutida considerando as duas definições. Destaca-se que o termo "previsão de impactos ambientais" é equivalente ao termo "riscos ambientais".

A Constituição Federal de 1988, em seu capítulo 4, artigo 225, § 1, inciso IV, exige estudo prévio de impacto ambiental para qualquer instalação de obra ou atividade potencialmente causadora de significativa degradação do meio ambiente (BRASIL, 1988). A Lei n. 6.938, de 31 de agosto de 1981, prevê que a avaliação de impactos ambientais é um dos instrumentos da Política Nacional do Meio Ambiente (BRASIL, 1981). 
A referida lei estabelece que, em uma avaliação de impactos ambientais, além de outros elementos, precisa ser gerado o Relatório de Impacto Ambiental (RIMA), que fornece todas as considerações sobre os possíveis impactos ambientais de um empreendimento, e o Estudo de Impacto Ambiental (EIA), que detalha toda a análise técnica feita pelos responsáveis que devem aprovar ou não o projeto. Com essa e outras leis, o governo demonstra a importância de uma avaliação de impactos ambientais bem realizada para a vida das pessoas.

Entre as técnicas para a avaliação de riscos/impactos ambientais, destaca-se a Matriz de Leopold, criada pelo geólogo Luna Bergere Leopold e seus colegas, em 1971, como uma resposta à Lei de Política Ambiental dos Estados Unidos de 1969, que não fornecia instruções claras de como gerar um relatório de análise de impactos ambientais para um projeto (JOSIMOVIC; PETRIC; MILIJIC, 2014).

A Matriz de Leopold permite uma visão geral e completa das ações de um projeto, das atividades impactantes que podem decorrer dele e das condições ambientais possivelmente atingidas, permitindo verificar quais as ações mais impactantes e quais as condições ambientais mais afetadas ou mesmo que podem vir a ser afetadas (ECONSERVATION, 2017). Esse instrumento permite que sejam previstos os impactos de um projeto, estabelecendo, assim, potenciais riscos no caso de sua implantação.

Por outro lado, o ritmo acelerado da informação na sociedade atual exige ferramentas rápidas, e os crescentes níveis de degradação do meio ambiente tornam urgente a criação de uma ferramenta rápida e eficiente para avaliar os impactos ambientais (STAMM, 2003). Por outro lado, o primeiro estudo para desenvolvimento de uma nova tecnologia corresponde à busca de anterioridade por meio de estudos prospectivos. Sua relevância está, entre outras, na eliminação de esforços e de energia destinados a algo que já foi desenvolvido, bem como na identificação de concorrentes e oportunidades de mercado.

Isso posto, com o propósito de desenvolver tecnologia para avaliação de riscos/impactos ambientais, baseada na Matriz de Leopold, este artigo tem como objetivo o estudo prospectivo, incluindo a elaboração da matriz SWOT (Strenghts, Weaknesses, Opportunities, Threats) e de Roadmap Tecnológico a serem utilizados na fase de desenvolvimento do projeto para visualização das tecnologias, produtos e mercados inter-relacionados.

\section{Metodologia}

A pesquisa realizada neste artigo tem uma abordagem quanti-qualitativa pelo fato de incluir dados quantitativos acerca dos pedidos de patentes e, também, os aspectos qualitativos relacionados à discussão sobre as patentes identificadas e suas relações com o produto a ser desenvolvido.

Por gerar conhecimento para aplicação em curto prazo, ou seja, desenvolvimento de ferramenta para avaliação de riscos/impactos, trata-se de uma pesquisa de natureza aplicada, também classificada como exploratória, dado que para sua concretização foram realizadas pesquisas na literatura científica e análises documentais e bibliográficas com a finalidade de elucidar as tecnologias relacionadas à avaliação de impactos ambientais, principalmente com o uso da Matriz de Leopold. 
Para prospecção tecnológica foram realizadas, a princípio, buscas de artigos científicos relacionados à avaliação de impactos ambientais que tinham como foco a Matriz de Leopold. Essa etapa foi realizada com buscas nos periódicos da CAPES (CAPES/MEC, 2020) pelo acesso CAFe, Scielo (SCIELO, 2020) e Scopus (ELSEVIER, 2020).

Em uma segunda etapa, foi realizada uma busca por aplicativos computacionais para avaliação de impactos ambientais em bases de dados oficiais do Instituto Nacional da Propriedade Industrial (INPI, 2020) e do Portal do Software público (GOVERNO DIGITAL, 2020). Como não existe obrigatoriedade no registro, também foram feitas pesquisas em bases de dados não oficiais, o "Source Forge"1 e o "Google Play Store"2. A seguir, na terceira etapa, o foco foi em patentes, por meio da prospecção tecnológica com busca nos bancos do Orbit (QUESTEL, 2020) e do INPI (INPI, 2020).

As palavras-chave utilizadas nas pesquisas foram: "Leopold Matrix", "Matrix" and "Leopold", "Analysis of environmental impact", "Assessment of Environmental impact", "Assessing environmental impact", "Environmental impact analysis" e "Environmental impact assessment". Todos os termos foram verificados no plural e no singular. Em bases nacionais também foram utilizados os termos em idioma Português, os quais neste artigo serão tratados como uma única consulta. Essas palavras foram escolhidas após a leitura de diversos artigos sobre o assunto, já que todos eles traziam pelo menos uma dessas palavras-chave em seus resumos.

$\mathrm{Na}$ quarta etapa, foi feita uma análise dos aspectos internos e externos que interferem na criação de um aplicativo para avaliação de impactos ambientais, com foco na Matriz de Leopold, por meio da Matriz SWOT (AKTOUF, 2002), sendo analisados fatores favoráveis e desfavoráveis do projeto.

Finalmente, com todos os dados obtidos nas etapas anteriores, foi confeccionado o Roadmap Tecnológico (BORSCHIVER; SILVA, 2016) que traça pontos importantes que nortearão o desenvolvimento da inovação proposta, gerando rentabilidade, dinamicidade, segurança $e$ motivação ao projeto.

\section{Resultados e Discussão}

Há uma grande quantidade de artigos científicos sobre avaliação de impactos ambientais de um modo geral (Tabela 1), já que "Environmental impact assessment" constitui um termo bastante abrangente. Isso também advém da importância do tema para a comunidade científica. Por outro lado, o número de artigos é considerado pequeno quando eles se referem à Matriz de Leopold, foco deste estudo, apontando a escassez de estudos relacionados à referida ferramenta. 
Tabela 1 - Busca por artigos científicos

\begin{tabular}{cccc} 
"Leopold Matrix" & CAPES & ScIELO & ScoPUS \\
"Matrix" and "Leopold" & 79 & 11 & 1 \\
"Analysis of environmental impact" & 378 & 11 & 1 \\
"Assessment of Environmental impact" & 78 & 976 & 184 \\
"Assessing environmental impact" & 155 & 486 & 552 \\
"Environmental impact analysis" & 1.068 & 94 & 277 \\
"Environmental impact assessment" & 120 & 976 & 1.360 \\
\hline
\end{tabular}

Fonte: Elaborada pelos autores deste artigo

Analisando os resumos dos trabalhos com foco na Matriz de Leopold, quase sua totalidade faz uso apenas da matriz para avaliação de impactos ambientais em um projeto específico. Uma pequena minoria argumenta uma forma mais eficiente de construir a matriz, sendo que esses trabalhos sugerem a utilização de planilha eletrônica para tal fim, chamada de "Ambitec" (GALHARTE; CRESTANA, 2007). Metade dos trabalhos utiliza a Matriz de Leopold na forma como foi concebida, e a outra metade busca adaptar a matriz para que ela consiga se aprofundar $e$ avaliar mais informações sobre os impactos ambientais de um projeto qualquer.

Entre os trabalhos que não possuem foco específico na Matriz de Leopold, muitos citam, a exemplo de Marañón, Mulgado e Rodríguez (2020) e outros, que até usam a matriz em algum momento do trabalho, contudo nenhum deles tem como objetivo a análise da matriz para sua maior eficiência e eficácia em processos de avaliação de impactos ambientais.

Nenhum resultado foi identificado na busca realizada no site do INPI (INPI, 2020) e nem no Portal do Software Público (GOVERNO DIGITAL, 2020) referente a programas de computador para Avaliação de Impactos Ambientais. Em todas as pesquisas, a mensagem foi sempre a mesma: "Nenhum resultado foi encontrado para a sua pesquisa" ou "0 Software(s)".

Esses resultados apontam o requisito de novidade da solução proposta, embora seja importante citar que o registro de software não é obrigatório, podendo então haver aplicativos não registrados no mercado, o que motivou a busca nas bases de dados não oficiais, o "Source Forge" e o "Google Play Store"4, sendo obtidos resultados somente a partir deste último (Tabela 2).

Tabela 2 - Consulta à base de dados do Google Play Store

\begin{tabular}{cc} 
"Leopold Matrix" & Google PLA \\
"Matrix" and "Leopold" & 0 \\
"Analysis of environmental impact" & 0 \\
"Assessment of Environmental impact" & 1 \\
"Assessing environmental impact" & 0 \\
"Environmental impact analysis" & 1 \\
"Environmental impact assessment" & 2 \\
\hline
\end{tabular}

Fonte: Elaborada pelos autores deste artigo 
Em uma análise criteriosa dos 61 resultados diferentes ${ }^{5}$, constatou-se que:

a) 55 resultados são apenas guias, roteiros, questionários e lembretes para estudantes e profissionais de várias engenharias, principalmente engenharia ambiental;

b) dois resultados são aplicações de marketing de empresas que prestam consultoria na área ambiental;

c) o aplicativo Environmental Inspection auxilia na realização de inspeções ambientais em um projeto. É apenas um cadastro e um checklist de elementos necessários para uma criteriosa inspeção ambiental;

d) o aplicativo Smart EIA identifica projetos que precisam de EIA (Estudo de Impacto Ambiental), conforme critérios adotados na Indonésia, país de origem do aplicativo;

e) o aplicativo WhaleReport é apenas para usuários informarem avistamentos de baleias, golfinhos, botos e tartarugas marinhas, com objetivo de ajudar um projeto ecológico na preservação desses animais; $e$

f) por fim, o aplicativo SCR-EHS auxilia empresas no processo de identificar, medir, controlar e melhorar os processos de negócios, incluindo fatores ambientais; na questão ambiental, esse aplicativo cria uma abordagem sistemática para o cumprimento das regulamentações ambientais.

Assim, a busca nas duas bases de dados de softwares não oficiais permite concluir que nenhum dos aplicativos encontrados na pesquisa tem a funcionalidade para realizar uma avaliação de impactos ambientais e muito menos se utiliza da Matriz de Leopold.

Na terceira etapa, relativa à prospecção tecnológica com busca por patentes, a partir da base de dados do INPI (INPI, 2020), foram encontradas 19 patentes. Entretanto, nenhuma delas refere-se à avaliação de impactos ambientais em projetos, muito menos menciona o uso da Matriz de Leopold. A totalidade das patentes encontradas referem-se à utilização de processos ou produtos para reduzir um impacto ambiental em uma situação específica de um projeto.

A busca utilizando o Orbit (QUESTEL, 2020) permitiu identificar também as patentes internacionais (Tabela 3), cujos resultados para as palavras-chave "Leopold Matrix" e "Matrix" and "Leopold", apesar de não terem trazido nenhum resultado em patentes, trouxeram 17 artigos científicos sobre o assunto (non-patent results no orbit), já identificados na primeira etapa de busca deste trabalho.

Tabela 3 - Consulta à base de dados do Orbit

\begin{tabular}{cc} 
"Leopold Matrix" & ORBIT \\
"Matrix" and "Leopold" & 0 \\
"Analysis of environmental impact" & 0 \\
"Assessment of Environmental impact" & 0 \\
"Assessing environmental impact" & 3 \\
"Environmental impact analysis" & 5 \\
"Environmental impact assessment" & 3 \\
\hline
\end{tabular}

Fonte: Elaborada pelos autores deste artigo

${ }^{5}$ Obteve-se um total de 62 resultados, porém um mesmo aplicativo apareceu em duas consultas diferentes, fazendo totalizar 61 aplicativos distintos. 
Destarte, foram encontradas dez patentes distintas, somando-se as buscas realizadas com as palavras-chave "Assessment of Environmental impact", "Assessing environmental impact" e "Environmental impact analysis" (Tabela 3). O total de 11 deve-se à repetição de uma das patentes. Entre estas, cinco encontram-se ativas/vivas e cinco inativas/mortas (QUESTEL, 2020).

Em relação às cinco patentes ativas, tem-se:

a) um método para analisar se os extratos contendo água são poluídos ou não por fraturamento hidráulico (JINXIU et al., 2018);

b) método ou processo para avaliar o impacto ambiental de um dispositivo de processamento durante seu ciclo de vida, com base nos dados de consumo (FRITZ, 2013);

c) sistema para avaliar o impacto ambiental do processamento de trabalhos de impressão (ARAZ; LEWIS; SUDHENDU, 2011);

d) avaliação do impacto ambiental do trânsito de veículos por meio de um dispositivo agregado ao veículo (CHRISTOPHER, 2015);

e) medidor de energia elétrica inteligente que acusa falhas e faz monitoramento das condições elétricas (DEZHONG; KAI, 2019).

As referidas patentes propõem avaliar ou minimizar o impacto ambiental em uma situação específica, não citando, de nenhuma forma, a Matriz de Leopold. Portanto, não tem relação direta com o processo de avaliação proposto neste trabalho, já que o foco é a avaliação de impactos ambientais de forma geral com o uso de uma Matriz de Leopold melhorada, incluindo critérios de grande relevância.

Devido ao aspecto abrangente relacionado ao termo "Environmental impact assessment" (última linha na Tabela 3), existem 105 famílias de patentes já depositadas. Destas, 67 (64\%) estão ativas, ou seja, concedidas ou em fase de análise (Gráfico 1).

Gráfico 1 - Situação das patentes contendo o termo "Environmental impact assessment" no título e/ou no resumo

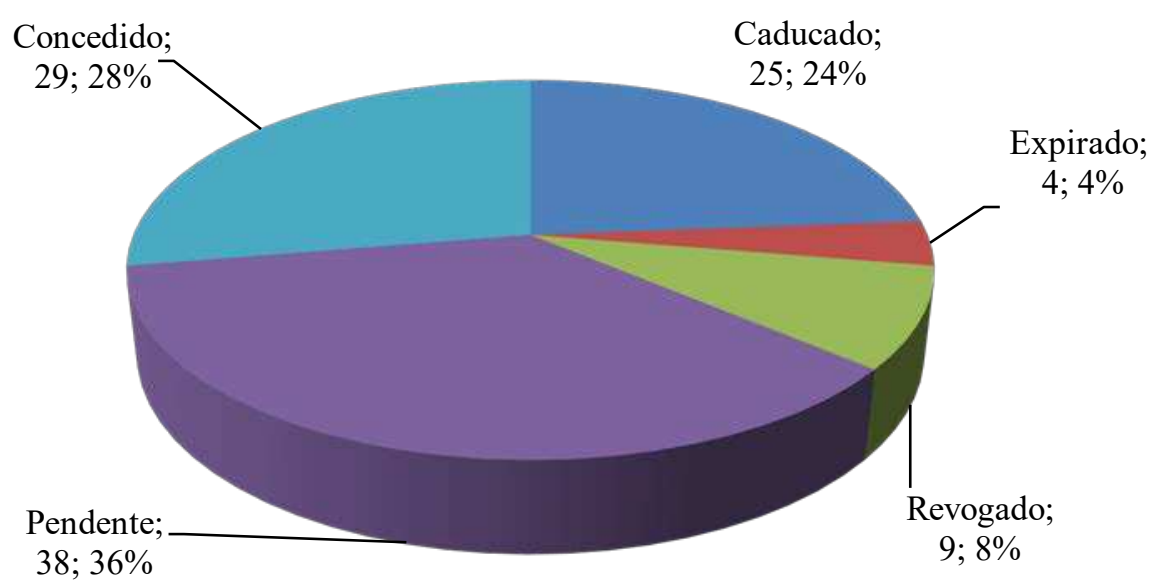

Fonte: Elaborado pelos autores deste artigo, a partir dos dados obtidos do Orbit (QUESTEL, 2020)

A maioria (74,28\%) foi depositada nos últimos dez anos (Gráfico 2), com nítido crescimento nos últimos cinco anos. Os pequenos quantitativos nos anos de 2019 e 2020 estão relaciona- 
dos ao período de sigilo das patentes, de modo que não podem ser utilizados para conclusões acerca de decréscimos, já que não incluem o quantitativo daquelas ainda não publicadas ainda.

Gráfico 2 - Ano de apresentação das patentes

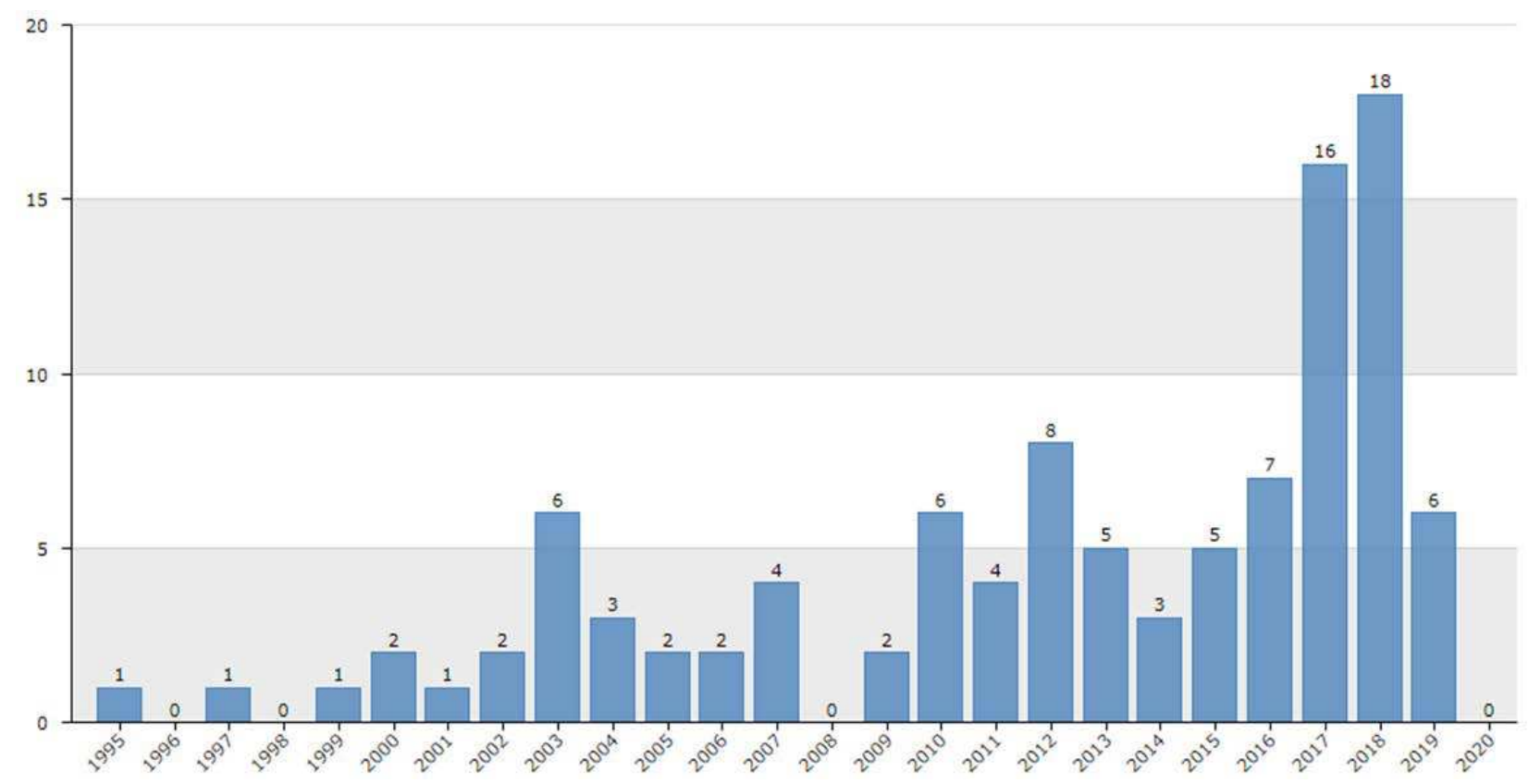

Fonte: Questel (2020)

Com relação ao desenvolvimento tecnológico na área de avaliação de impactos ambientais no mundo, destaca-se a China, com 47 famílias de patentes, seguida pelo Japão e Coreia do Sul. Na América, somente nos Estados Unidos há patentes ativas, sendo, no caso, três famílias (Gráfico 3). Em números menos expressivos, registra-se a Índia (IN), Organização Mundial da Propriedade Intelectual (WO), Áustria (AU) e Organização Europeia de Patentes (EP).

Gráfico 3 - Número de patentes ativas na área de avaliação de impactos ambientais por país

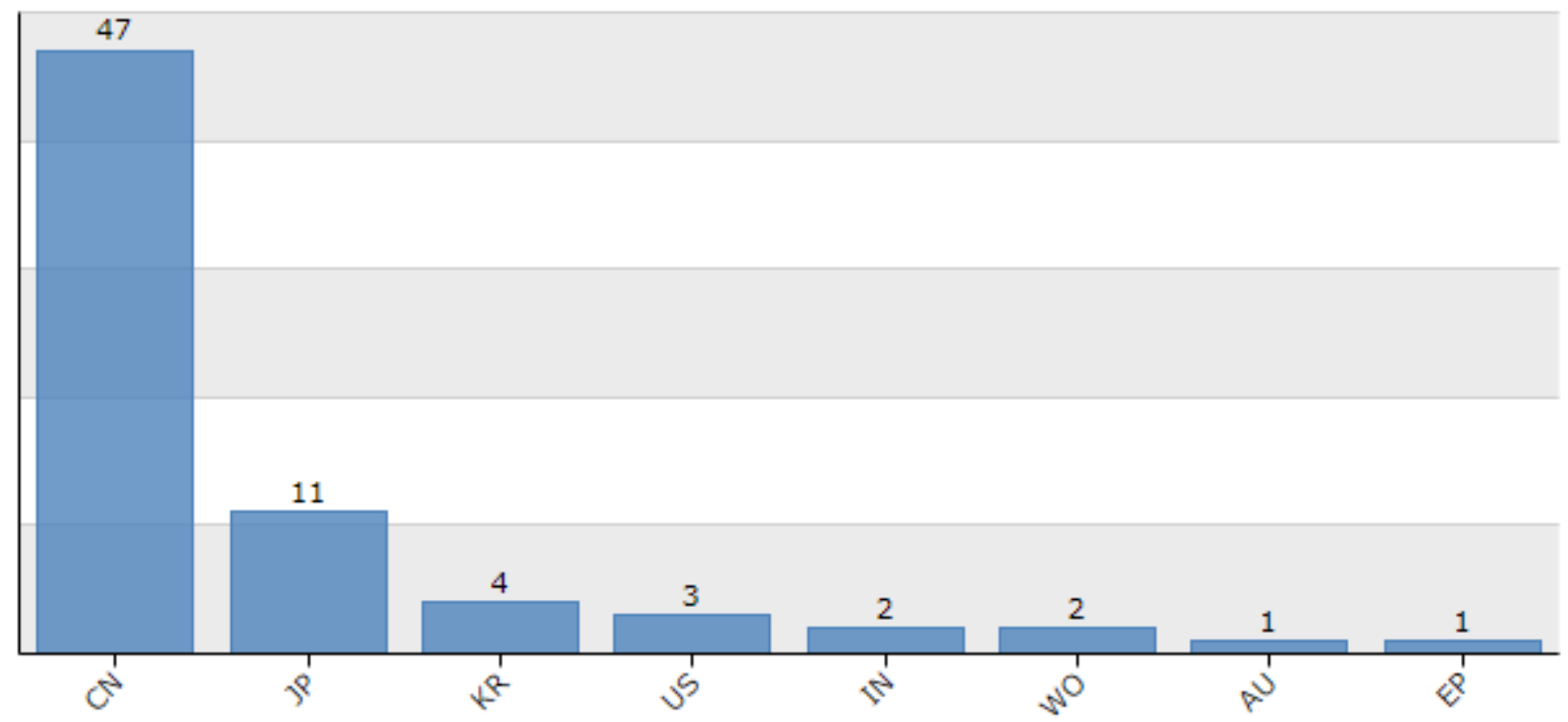

Fonte: Questel (2020) 
Com relação à avaliação de impactos ambientais, as patentes ativas estão majoritariamente no domínio tecnológico dos Métodos de Tecnologia da Informação aplicados ao gerenciamento. Ademais a classificação em vários outros domínios, além das tecnologias da computação, como transporte; química dos materiais e dos alimentos; engenharia civil; engenharia química; motores, bombas e turbinas; e biotecnologia (Figura 1), apontam o caráter interdisciplinar do tema proposto.

Figura 1 - Domínios tecnológicos das patentes ativas na área de avaliação de impactos ambientais

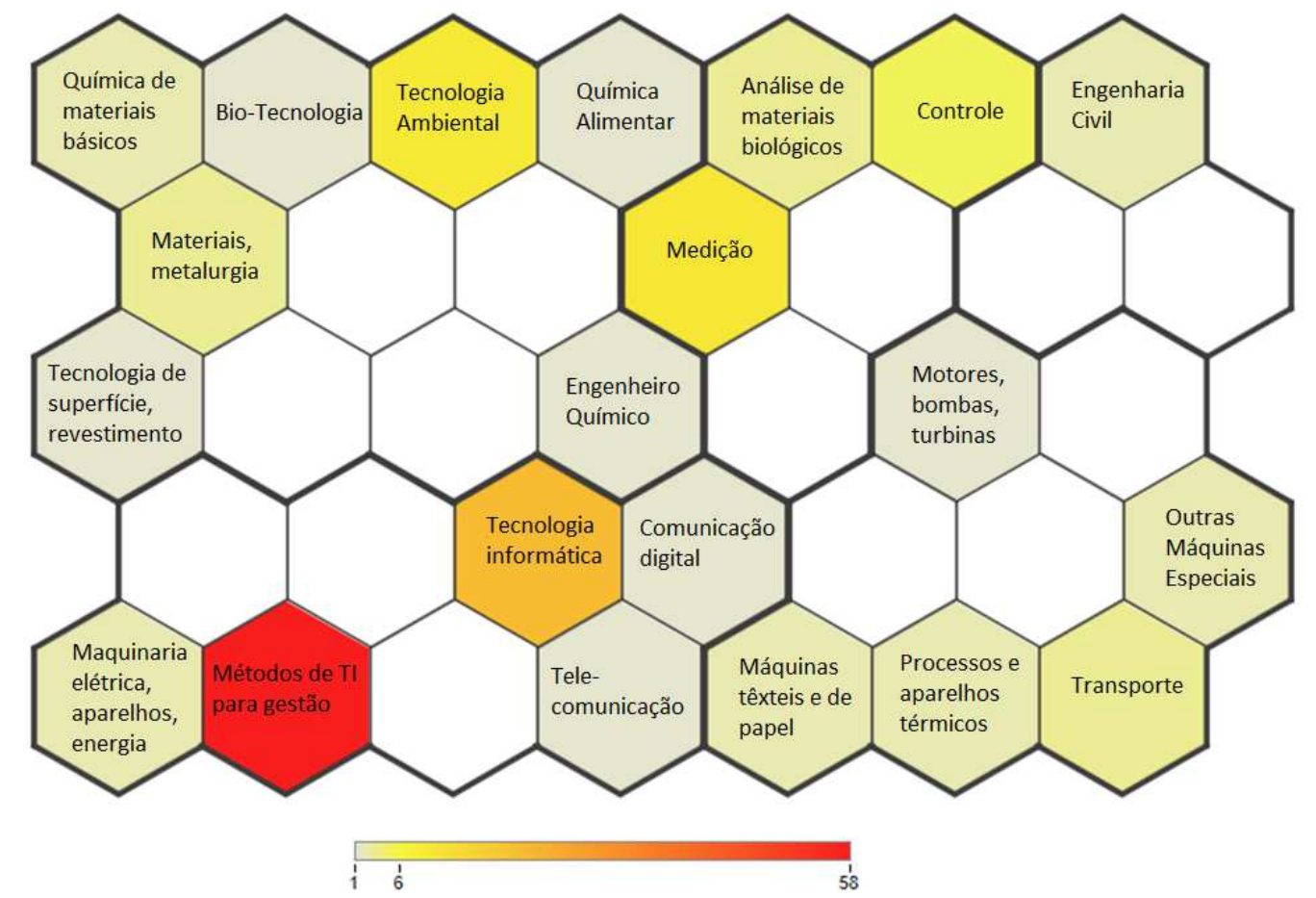

Fonte: Questel (2020)

Informações adicionais foram obtidas a partir da análise detalhada acerca das referidas patentes ativas, as quais estão descritas a seguir e sumarizadas no Gráfico 4:

a) 32 referem-se a técnicas ou ferramentas de avaliação de impactos ambientais de uma atividade impactante específica;

b) 15 referem-se a técnicas ou ferramentas de avaliação de impactos ambientais de uma única condição ambiental específica, tal como a água, o ar, o ruído, ou outros;

c) sete são técnicas ou ferramentas apenas para organização de relatórios e dados ou auxílio aos profissionais que realizam avaliações de impactos ambientais;

d) 13 são técnicas ou ferramentas voltadas para a avaliação de impactos ambientais de um projeto qualquer:

i. duas com base no passo a passo do ciclo de vida de um projeto;

ii. quatro com base em um sistema de pontuação, atribuindo pontos à cada atividade impactante;

iii. uma com base na fabricação de produtos;

iv. três com base em comparação de dados armazenados do ambiente sem atividades impactantes;

v. três com base em cálculos matemáticos de dados a partir das atividades impactantes. 
Assim, conclui-se que existem poucas tecnologias para a avaliação de impactos ambientais. Apesar de concorrentes, as famílias de patentes de projetos que avaliam impactos ambientais não refletem o projeto aqui proposto, pois nenhuma delas realiza a avaliação de impactos ambientais com base na Matriz de Leopold, porém, o produto proposto pode vir a somar esforços com projetos já existentes e protegidas por patentes para uma visão ainda mais ampla ou complementar dos impactos ambientais, permitindo uma avaliação ainda mais segura para os tomadores de decisão.

Gráfico 4 - Foco de aplicação das famílias de patentes ativas

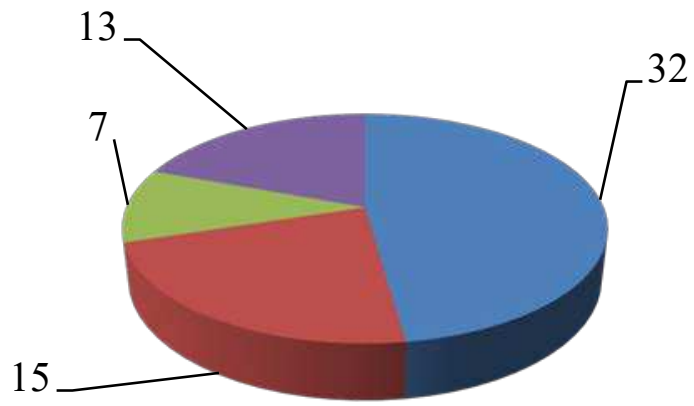

- Atividade Impactante Específica

- Condição Ambiental Específica

- Auxílio aos profissionais

- Avaliação de Impactos Ambientais

Fonte: Elaborado pelos autores deste artigo

Adicionalmente, por meio da Matriz SWOT, são facilmente visualizados os fatores internos e externos que influenciam a inserção do projeto tecnológico proposto no mercado, permitindo planejamento $e$ ações que minimizam fraquezas $e$ ameaças e que potencializem as forças $e$ oportunidades, amparando ou viabilizando o desenvolvimento (Quadro 1). Em suma, a análise da matriz auxiliou na elaboração de estratégias importantes para o planejamento do projeto.

Quadro 1 - Análise dos fatores internos e externos (Matriz SWOT) ao desenvolvimento do produto proposto neste estudo

\begin{tabular}{|c|c|c|}
\hline & FAVORÁvel & DESFAVORÁvel \\
\hline & Forças & Fraquezas \\
\hline \multirow[t]{2}{*}{ Interno } & $\begin{array}{c}\text { Equipe interdisciplinar com mão de } \\
\text { obra qualificada, inclusive na área } \\
\text { de desenvolvimento de sistemas. } \\
\text { Infraestrutura que atende aos } \\
\text { requisitos do projeto. } \\
\text { Ambiente favorável de desenvolvimento. }\end{array}$ & $\begin{array}{c}\text { Equipe reduzida. } \\
\text { Equipe não familiarizada com os } \\
\text { detalhes da pesquisa. } \\
\text { Possível rotatividade de membros da equipe. }\end{array}$ \\
\hline & Oportunidades & Ameaças \\
\hline Externo & $\begin{array}{c}\text { Não encontrado sistemas } \\
\text { concorrentes similares. } \\
\text { Produto flexível para atuar } \\
\text { em diversas áreas. } \\
\text { Projeto pode ser executado em } \\
\text { diferentes plataformas. } \\
\text { Uso simples e com resultados validados. } \\
\text { Momento político-econômico nacional. }\end{array}$ & $\begin{array}{l}\text { Cópias não autorizadas. } \\
\text { Roubo da ideia, plágio. } \\
\text { Surgimento de tecnologia concorrente } \\
\text { durante a fase de desenvolvimento. } \\
\text { Pouco apoio financeiro. } \\
\text { Parte do público alvo não se interessar } \\
\text { pela inovação, resistência. } \\
\text { Momento político-econômico nacional. }\end{array}$ \\
\hline
\end{tabular}

Fonte: Elaborado pelos autores deste artigo 
A fraqueza quanto à equipe reduzida (Quadro 1) é amenizada pelas habilidades dos seus membros para o desenvolvimento de novas tecnologias, bem como devido ao fato de constituir atividade prevista nas suas obrigações para com o cargo, no caso dos docentes, ou entre os requisitos para obtenção do título de doutor, no caso de discente de pós-graduação, com tempo estabelecido para o desenvolvimento da tecnologia. Acredita-se que a rotatividade da equipe será próxima de zero. Apesar do largo tempo, a equipe está engajada e motivada a colocar o produto no mercado e proteger a tecnologia por meio de patente de processo, a fim de evitar o uso indevido da tecnologia proposta.

Para minimizar ameaça de cópias (Quadro 1 - Ameaças) ou usos não autorizados, apropriação indevida da ideia e plágio, estão em trâmites internos na Universidade Federal do Vale do São Francisco (UNIVASF) o pedido de proteção por patente do processo de avaliação implementado por programa de computador, o registro da marca que atribui elementos distintivos à solução proposta e, finalmente, o registro do código-fonte. Ademais, após a finalização dos trâmites para proteção da tecnologia, será elaborada e executada uma campanha de marketing como canal de acesso à tecnologia a partir do interesse do público. Essa campanha será baseada em redes sociais a partir de comunicação e de aprovação do Núcleo de Inovação Tecnológica da UNIVASF e, também, será adicionada à vitrine tecnológica da Instituição.

Apesar da inexistência de apoio financeiro externo (Quadro 1 - Ameaças), a equipe dispõe do capital mínimo necessário para a execução do projeto, desde sua concepção e em todas as fases de seu desenvolvimento e implantação. A despeito do momento político-econômico que passa por dificuldades, devido à pandemia e à recessão, existe um forte estímulo à digitalização dos processos e à inovação, o que abre um horizonte de vantagens para a disseminação do produto no mercado.

Após levantamento acerca do que já existe no mercado ou apenas em patentes, bem como a confirmação da inexistência de produtos ou processos protegidos por meio de patentes similares à proposta desta pesquisa, pode-se obter o Roadmap Tecnológico (Tabela 4), que auxilia no direcionamento do projeto para preencher as lacunas existentes, observar o mercado e focar o planejamento futuro. Com relação às concorrentes diretas da tecnologia proposta, destacam-se aquelas que são objetos de famílias de patentes vivas ou ativas e que promovem técnicas ou aplicações para avaliação de impactos ambientais de maneira geral e também a planilha "Ambitec", mencionada na primeira etapa deste estudo.

Para execução do projeto da tecnologia implementada por meio de programa de computador proposta nesta pesquisa no seu planejamento em curto prazo, faz-se necessária a escolha da linguagem de programação para desenvolvimento da versão desktop e, em médio prazo, para desenvolvimento da versão Android. Finalmente, em longo prazo, são usados notebooks ou celulares ou mesmo desktop para validação da tecnologia (análises feitas em campo). 
Com relação ao mercado consumidor, o foco inicial de aplicação da tecnologia proposta está no auxílio à tomada de decisão a partir dos gestores de projetos agroecológicos e de desenvolvimento territorial. Sua versatilidade de aplicação permite sua expansão para outros gestores, como aqueles de projetos rurais e também aqueles responsáveis por quaisquer processos de produção em geral. Esse foco em projetos agroecológicos e de desenvolvimento territorial resulta da urgência em verificar se esses projetos realmente atendem às necessidades naturais do meio ambiente, minimizando ou até eliminando impactos ambientais negativos.

Verifica-se que, em curto prazo, há concorrentes (Tabela 4). Entretanto, devido à pesquisa estar direcionada à aplicação em projetos agroecológicos e de desenvolvimento territorial, esses concorrentes podem ser desconsiderados no período, dada a não previsão de atuação das tecnologias concorrentes nos referidos projetos. Outra vantagem está no fato de que os concorrentes são, em quase sua totalidade, asiáticos, sem patente depositada no Brasil, de modo que se supõe que os titulares das referidas patentes não têm o Brasil como mercado em potencial. Com relação ao único concorrente nacional, é importante destacar que a tecnologia proposta não constitui um aplicativo e não possui as facilidades de uso da tecnologia aqui proposta.

Em médio prazo, com o sistema lançado e após os testes em projetos agroecológicos e de desenvolvimento territorial, o sistema está preparado para ser utilizado em projetos rurais de qualquer natureza. Novos concorrentes poderão estar no mercado, pois alguns estão com a patente pendente e poderão ter sido concedidas.

Em longo prazo, a previsão de lançamento de cartilhas com as experiências do uso da ferramenta em campo contribui para a orientação aos avaliadores de impactos ambientais, no sentido de facilitar ainda mais a adesão dos interessados pela tecnologia, bem como seu uso correto e confiável.

Finalmente, o estudo prospectivo, seguido pela análise SWOT, permite a elaboração de um Roadmap Tecnológico com ações e prazos para minimização de fraquezas e ameaças, constituindo um aparato que reduz a escolha de caminhos inoportunos, apresenta necessidades $e$ favorece a definição de metas e de possíveis desdobramentos do projeto. 
Quadro 2 - Roadmap Tecnológico

\begin{tabular}{|c|c|c|c|c|}
\hline & & CURTO PRAZO & MÉDIO PRAZO & LONGO PRAZO \\
\hline & Toshiba - Concedida & JP2007072708 A & & \\
\hline & Toray Industries - Concedida & JP2011186798 A & & \\
\hline & Hitachi - Concedida & JP2004265012 A & & \\
\hline & Bionova - Pendente & & $\begin{array}{l}\text { US20180357144 } \\
\text { A1 }\end{array}$ & \\
\hline & Toshiba - Concedida & JP2011203832 A & & \\
\hline 屴 & $\begin{array}{l}\text { Green Light Environment - } \\
\text { Concedida }\end{array}$ & WO201649697 A1 & & \\
\hline$\underset{\sim}{\dddot{\alpha}}$ & Hitachi - Concedida & JP2005327182 A & & \\
\hline $\begin{array}{l}\frac{1}{\alpha} \\
\text { O } \\
0\end{array}$ & $\begin{array}{l}\text { Guangzhou Xiaopeng Motors } \\
\text { Technology } 0 \text { - Pendente }\end{array}$ & & CN110053629 A & \\
\hline ¿̨ & $\begin{array}{c}\text { Nippon Telegraph \& Telephone - } \\
\text { Concedida }\end{array}$ & JP2015228196 A & & \\
\hline 운 & $\begin{array}{c}\text { Nippon Telegraph \& Telephone } \\
\text { - Concedida } \\
\end{array}$ & JP2014035725 A & & \\
\hline $\begin{array}{l}\text { O } \\
\stackrel{\sim}{\Psi} \\
\Sigma\end{array}$ & $\begin{array}{c}\text { Wuhan Xinjiangcheng } \\
\text { Environmental Affairs Consulting } \\
- \text { Pendente }\end{array}$ & & CN110348738 A & \\
\hline & TCO2 - Concedida & JP2017068362 A & & \\
\hline & $\begin{array}{c}\text { North China Electric Power } \\
\text { University - Pendente }\end{array}$ & & CN106408214 A & \\
\hline & $\begin{array}{c}\text { EMBRAPA - Ferramenta } \\
\text { Livre/Gratuita } \\
\text { Não patenteada }\end{array}$ & AMBITEC & & \\
\hline & $\begin{array}{l}\text { Projetos de Patentes } \\
\text { Caducadas/Revogadas }\end{array}$ & & & DIVERSAS \\
\hline 웅 & & $\begin{array}{c}\text { De projetos } \\
\text { Agroecológicos e } \\
\text { de Des. Territorial }\end{array}$ & & \\
\hline 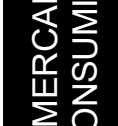 & Tomadores de decisão & & $\begin{array}{l}\text { De projє } \\
\text { gropecu } \\
\text { tradicior }\end{array}$ & $\mathbb{y}$ \\
\hline$<0$ & & & & $\begin{array}{l}\text { De projetos em } \\
\text { Geral } \\
\end{array}$ \\
\hline & Artigos/Publicações & Prospecção/Teoria & & Prática \\
\hline (D) & $\begin{array}{c}\text { Software versão desktop para } \\
\text { AIA }\end{array}$ & & Programa SAIA & \\
\hline$\stackrel{5}{\frac{5}{2}}$ & $\begin{array}{c}\text { Software versão android para } \\
\text { AIA }\end{array}$ & & $\mathrm{A} \backslash$ & \\
\hline $\begin{array}{l}\bar{O} \\
\stackrel{\leftrightarrow}{a}\end{array}$ & $\begin{array}{c}\text { Estudo sobre a criação e o uso } \\
\text { do aplicativo }\end{array}$ & & & $\begin{array}{c}\text { TESE } \\
\text { Cartilha de } \\
\text { Uso/Tutorial }\end{array}$ \\
\hline & Registro & 7 & INPI/ Patente & 7 \\
\hline$\frac{4}{0}$ & $\begin{array}{l}\text { Sistemas de Buscas de } \\
\text { Artigos/Patentes }\end{array}$ & $\begin{array}{c}\text { Orbit } \\
\text { INPI } \\
\text { Periódicos CAPES } \\
\text { Scielo } \\
\text { Scopus } \\
\end{array}$ & & \\
\hline Oे & Linguagens de Programação & Delphi & $\begin{array}{r}\text { Java } \\
\text { JavaScript } \\
\text { AndroidStudio }\end{array}$ & \\
\hline 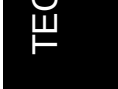 & $\begin{array}{c}\text { Outros elementos } \\
\text { computacionais }\end{array}$ & Bases de Dados & & $\begin{array}{c}\text { Editores de } \\
\text { Texto/Gráficos } \\
\end{array}$ \\
\hline & Dispositivos Móveis & & & $\begin{array}{c}\text { Celular Android } \\
\text { Notebook } \\
\text { Windows }\end{array}$ \\
\hline
\end{tabular}

Fonte: Elaborado pelos autores deste artigo 


\section{Considerações Finais}

À medida que a população humana cresce exponencialmente e se intensificam os efeitos da necessidade de produção em grande escala, a conservação do meio ambiente tem caráter urgente e inadiável, cabendo aos novos profissionais de quaisquer áreas a realização de estudos de avaliação dos impactos ao meio ambiente causados por suas atividades. Esses fatores evidenciam a importância de um projeto capaz de apresentar e de compilar dados de atividades impactantes de maneira ágil e precisa, fornecendo, de maneira completa e simplificada, todos os elementos relevantes para que seja feita uma tomada de decisão com base em todos os ângulos ambientais que a natureza precisa.

A Matriz SWOT permitiu identificar as oportunidades e forças para potencializá-las, bem como as fraquezas a serem minimizadas, sendo possível destacar que, inicialmente, contava-se com uma pequena equipe ainda não familiarizada com os resultados da pesquisa, o que permitiu a análise e a conclusão de que seria superada pelas habilidades inerentes aos membros e diante de um planejamento rigoroso com relação à dedicação da equipe. Com relação às ameaças externas relativas à ameaça de ou usos não autorizados e à apropriação indevida da ideia e plágio, foi identificada a necessidade de proteção, por meio de patente, do registro de programa de computador e de marca.

Os possíveis concorrentes descritos no Roadmap Tecnológico, nas distintas fases do desenvolvimento, com tecnologias patenteados ou não, que foram analisadas em comparação à nossa proposta, não possuem similaridade com nosso projeto, evidenciando o caráter inovador da solução e seu diferencial no mercado nacional.

Este estudo compõe uma demanda de produto de interesse acadêmico e de mercado para a agroecologia e o desenvolvimento territorial que pode auxiliar não somente os tomadores de decisão, como também pesquisadores de atividades impactantes. Nesse sentido, conclui-se pelo aspecto promissor da tecnologia, seu carácter inovador e impactante, trazendo benefícios ao meio ambiente, com repercussões positivas para o desenvolvimento econômico sustentável e o bem-estar social.

\section{Perspectivas Futuras}

Para trabalhos futuros, sugere-se que sejam realizadas pesquisas para o desenvolvimento de planos de comunicação entre os principais envolvidos em processos de avaliação, tal como os consultores em meio ambiente, atores de sistemas agroecológicos e agentes públicos para autorização da implementação e funcionamento de indústrias, bem como pesquisas para o desenvolvimento de outras tecnologias de avaliação de impactos ambientais, com implementação de outros métodos complementares de avaliação de impactos, gerando tecnologias derivadas desta ou de novas tecnologias, sendo sugeridas as redes de interação, com visualização de impactos primários, secundários e terciários e/ou o método de sobreposição de mapas. 


\section{Referências}

AKTOUF, O. Governança e pensamento estratégico: uma crítica a Michel Porter. RAE - Revista de Administração de Empresa, São Paulo, v. 42, n. 3, p. 43-57, 2002. ISSN 2178-938X.

ARAZ, Khachatrian; LEWIS, S. Snow; SUDHENDU, Rai. System for assessing environmental impact of processing print jobs. A1. Data da $1^{\text {a }}$ Prioridade: 16 jun. 2011. US20110145085.

BATISTA, Ivaniza S. et al. Avaliação de Impactos Ambientais. In: XVII SIMPÓSIO BRASILEIRO DE GEOGRAFIA FÍSICA APLICADA, Campinas, 2017, p. 7.188-7.199. Anais [...]. Campinas, 2017.

BORSCHIVER, S.; SILVA, A. L. R. Technology roadmap: Planejamento Estratégico para alinhar Mercado-Produto-Tecnologia. Rio de Janeiro: Interciência, 2016.

BRASIL. Constituição da República Federativa do Brasil. Brasília, DF: Senado Federal, 1988.

BRASIL. Lei n. 6.938, de 31 de agosto de 1981. Dispõe sobre a Política Nacional do Meio Ambiente, seus fins e mecanismos de formulação e aplicação, e dá outras providências. Disponível em: http://www.planalto.gov.br/ccivil_03/leis/16938.htm. Acesso em: 6 maio 2021.

CAPES/MEC. Portal de Periódicos CAPES/MEC. Portal de Periódicos CAPES/MEC, 2020. Disponível em: https://www.periodicos.capes.gov.br/. Acesso em: 2 abr. 2020.

CHRISTOPHER. Scofield. Assessing environmental impact of vehicle transit. Data da $1^{\text {a }}$ Prioridade: 27 fev. 2015. EP3113998.

CONAMA - CONSELHO NACIONAL DO MEIO AMBIENTE. Resolução do Conselho Nacional do Meio Ambiente N. 001. Diário Oficial da União, Brasília, DF, 17 fev. 1986.

DAGNINO, Ricardo de Sampaio; CARPI JUNIOR, Salvador. Risco Ambiental: Conceitos e Aplicações. Climatologia e Estudos da Paisagem, Rio Claro, v. 2, n. 2, p. 50, 2007. ISSN 1980 $654 \mathrm{X}$.

DEZHONG, Ma; KAI, Wang. Intelligent electric energy meter failure mechanism and condition monitoring system and method. Data da $1^{\text {a }}$ Prioridade: 27 ago. 2019. CN110531302.

ECONSERVATION. Avaliação dos Impactos Ambientais. Vitória: Econservation, Estudos e Projetos Ambientais., 2017. (RT ECV 057/17).

ELSEVIER. Scopus. Scopus Preview, 2020. Disponível em: https://www.scopus.com/. Acesso em: 2 abr. 2020.

ENRÍQUEZ-DE-SALAMANCA, Álvaro. Stakeholders' manipulation of Environmental Impact Assessment. Environmental Impact Assessment Review, Amsterdã, v. 68, p. 10-18, jan. 2018. ISSN 0195-9255.

FRITZ, F. Ebner. Method and system for assessing environmental impact of a processing device. A1. Data da $1^{\text {a }}$ Prioridade: 18 abr. 2013. US20130096893.

GALHARTE, Caroline Alves; CRESTANA, Silvio. Avaliação de Impactos Ambientais da integração lavoura-pecuária: Estudo de Caso da inovação tecnológica da EMBRAPA. 2007. 121p. Dissertação (Mestrado) - Escola de Engenharia de São Carlos, São Carlos, 2007.

GOVERNO DIGITAL. Software Público, 2020. Disponível em: https://www.gov.br/governodigital/ pt-br/software-publico. Acesso em: 2 abr. 2020. 
HIDEKI, Noda et al. Environmental impact assessment system and environmental impact assessment method. Data da $1^{\text {a }}$ Prioridade: 6 set. 2005. JP2007072708A.

HIDEKI, Noda; TAKENORI, Kobayashi. Environmental impact assessment system, environmental impact assessment method and environmental impact assessment program. Data da $1^{\text {a }}$ Prioridade: 24 mar. 2010. JP2011203832A.

INPI - INSTITUTO NACIONAL DA PROPRIEDADE INDUSTRIAL. Site do Instituto Nacional da Propriedade Industrial/Ministério da Economia, 2020. Disponível em: http://www.inpi.gov.br/. Acesso em: abr. 2020.

JAY, Stephen et al. Environmental impact assessment: Retrospect and prospect. Environmental Impact Assessment Review, Amsterdã, p. 287-300, maio de 2007.

JINXIU, Yang et al. Method for analyzing whether water-bearing strata are polluted by hydraulic fracturing or not by aid of gas and liquid mixed tracers. A. Data da $1^{\text {a }}$ Prioridade: 30 nov. 2018. CN108915674.

JOSIMOVIC, B.; PETRIC, J.; MILIJIC, S. The Use of the Leopold Matrix in Carrying Out the EIA for Wind Farms in Serbia. Energy and Environment Research, Richmond Hill, v. 4, n. 1, 2014. ISSN 1927-0577.

JUAN, Li. Interactive control method and device, electronic equipment and storage medium. Data da $1^{\text {a }}$ Prioridade: 7 maio 2019. CN110053629A.

KAZUYA, Matsumura; OSAMU, Mito. Environmental impact assessment system, environmental impact assessment program and recording medium. Data da $1^{\text {a }}$ Prioridade: 09 mar. 2010. JP2011186798A.

KOICHI, Shobatake. Information search method, information search program, and information search system. Data da $1^{\text {a }}$ Prioridade: 28 set. 2015. JP2017068362A.

LIU, Yang. Hospital environmental influence factor assessment method. Data da $1^{\text {a }}$ Prior.:12 jul. 2019. CN110348738A.

MARAÑÓN, Alina González; MULGADO, Israel Palacios; RODRÍGUEZ, Arelis Ábalos. Impacto ambiental del vertido de residuales en la cuenca hidrográfica Guaos-Gascón de Santiago de Cuba. Revista Cubana de Química, Santiago de Cuba, v. 32, n. 1, 2020.

MMA - MINSTÉRIO DO MEIO AMBIENTE. Caderno de Licenciamento Ambiental. Brasília, DF: MMA, 2009.

PANU, Pasanen. Computer implemented method for generating sustainable performance and environmental impact assessment for target system. Data da $1^{\text {a }}$ Prioridade: 8 jun. 2017. US20180357144A1.

PETRINA, Raitt. A computing device, computer implemented method and computer readable storage medium for the generation of hierarchical data structure environmental impact assessment reports. Data da $1^{\text {a }}$ Prioridade: 30 set. 2014. WO201649697A1.

PIMENTEL, G.; PIRES, S. H. Metodologias de avaliação de impacto ambiental: aplicações e seus limites. Revista de Administração Pública, Rio de Janeiro, p. 56-68, 1992.

QUESTEL. Orbit Intelligence. Orbit Intelligence. [2020]. Disponível em: https://www.orbit.com/. Acesso em: 2 abr. 2020. 
SANGUINETTO, Evandro. Avaliação de Impactos Ambientais (AIA), Avaliação Ambiental Estratégica (AAE) e Sustentabilidade em Minas Gerais. Revista Labor \& Engenho, Campinas, v. 5, n. 3, p. 100-120, 2011. ISSN 2176-8846.

SATORU, Ishida. Environmental impact assessment system. Data da $1^{\text {a }}$ Prioridade: 17 maio. 2004. JP2005327182A.

SATORU, Ishida; SASAKI, Tasayo; MIYUKI, Kawada. Environmental impact assessment method and environmental impact assessment apparatus. Data da $1^{\text {a }}$ Prioridade: 28 fev. 2003. JP2004265012A.

SCIELO. SciELO - Scientific Electronic Library Online. SciELO - Scientific Electronic Library Online, 2020. Disponível em: https://scielo.org/. Acesso em: 2 abr. 2020.

SHINSUKE, Iihashi; YUICHIRO, Takei; TOMOMI, Nagao. Environmental impact assessment system and method. Data da $1^{\text {a }}$ Prioridade: 3 jun. 2014. JP2015228196A.

STAMM, Hugo Roger. Método para Avaliação de Impacto Ambiental (AIA) em projetos de grande porte: Estudo de caso de uma usina termelétrica. 2003. 284p. Tese (Doutorado em Engenharia de Produção) - Programa de Pós-Graduação em Engenharia de Produção, Universidade Federal de Santa Catarina, Florianópolis, 2003.

TOMOMI, Nagao et al. Ict solution environmental impact assessment system and method. Data da $1^{\text {a }}$ Prioridade: 10 ago. 2012. JP2014035725A.

WEIJUN, Wang et al. Equivalent conversion evaluation method for full-life-cycle environmental cost of project. Data da $1^{\text {a }}$ Prioridade: 28 out. 2016. CN106408214A.

\section{Sobre os Autores}

\section{Roberto Tenório Figueiredo}

E-mail: tenorio.petrolina@bol.com.br ORCID: http://orcid.org/0000-0002-4753-8989

Mestre em Ciência da Computação pela Universidade Federal de Pernambuco em 2014.

Endereço profissional: FACAPE - Faculdade de Petrolina, Campus Universitário, s/n, Vila Eduardo, Petrolina, PE. CEP: 56328-903.

\section{Vivianni Marques Leite dos Santos}

E-mail: vivianni.santos@gmail.com ORCID: http://orcid.org/0000-0001-8741-8888

Doutora em Química pela Universidade Federal de Pernambuco em 2004.

Endereço profissional: UNIVASF Campus Juazeiro, Av. ACM, n. 510, Santo Antônio, Juazeiro, BA. CEP: $48902-300$.

\section{Jorge Luis Cavalcanti Ramos}

E-mail: jorge.cavalcanti@univasf.edu.br ORCID: http://orcid.org/0000-0002-6099-6861

Doutor em Ciência da Computação pela Universidade Federal de Pernambuco em 2016.

Endereço profissional: UNIVASF Campus Juazeiro, Av. ACM, n. 510, Santo Antônio, Juazeiro, BA. CEP: $48902-300$. 